\title{
Design and Simulation of a certain type Gun Interactive Virtual Maintenance Platform
}

\author{
LI Hua a , WANG Lei, SU Zhong-ting ${ }^{\text {b }}$, YANG Ming-hua \\ (Department of Arms and Control, Academy of Army Armored Force, Beijing, 100072, China) \\ aiuanne@163.com, bsuzt1984@163.com
}

Keywords: gun; virtual maintenance; Ngrain; PERT

Abstract: Aiming at the problems such as limited time, limited arms, limited tools, hard to faults simulation show and hard to speek the fault test theory and maintenance process, this paper designed the gun interactive virtual maintenance platform and analyzed the fault simulation. First of all, the three deriction virtual maintenance training environment is built up and the space is partitioned by the function, then the three-dimensional entity model and the three-dimensional cartoon are introduced, the gun virtual maintenance process is described by designing PERT figures, the basic maintenance job is simulated based on the interactive behavior model, lastly, the simulation cartoon and virtual maintenance operation flow are controlled interactively, the gun interactive virtual maintenance training in the three-dimensional environment is achieved, the gun maintenance training level is improved and the original and effective technical method is afforded for arms structure and maintenance teaching and training.

\section{Introduction}

A certain type parallel machine gun is a kind of little bore machine gun installed at the side of tank artillery which is used for destroy and suppress the infantry, antitank weapons and light-weight armored target of hostile forces ${ }^{[1]}$. The armored vehicle allocated parallel machine gun is very common and widely used. The structure of parallel machine gun is very complex and the assembly relationship is very accurate although the volume is very small ${ }^{[2]}$. Any fault component will lead to the abnormal firing of parallel machine gun. The parallel machine gun is used frequently in the training and easy to give rise to fault ${ }^{[3]}$. And the problems such as limit using time, wear and tear, limit equipment, hard to simulate and demonstrate of real fault and hard to explain the fault test theory and service process are ubiquitous which lead to the service training poor effect and big equipment wear and tear ${ }^{[4]}$.

To solve these problems, this paper designs the parallel machine gun interactive virtual maintenance system, built up and optimize the three-dimensional model in the virtual environment taking Ngrain software as programming development tool. The PERT chart describes the parallel machine gun virtual maintenance process, simulate the basic maintenance work based on the interactive behavior model and interactively control the simulation animation virtual maintenance operating process which can realize the parallel machine gun interactive virtual maintenance training in the three-dimensional environment and separate the subjects from the traditional and uninteresting training pattern. The deficiency of real training is made up and the parallel machine gun maintenance training level is improved which afford a new technical method for vehicle weapon structure and maintenance teach and training. 


\section{System design thinking}

The virtual maintenance training platform is designed according to the parallel machine gun maintenance scheme, maintenance outline and maintenance fingerpost combining the army maintenance training requirement investigate and the weakness of real maintenance which is shown as fig.1.

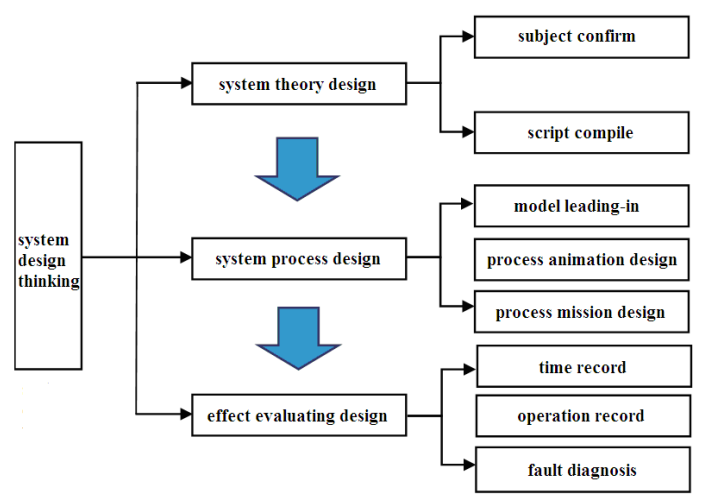

Fig.1 the design thinking of system

The work flow path of system is that the training subject is chosen according to the real need, then the parallel machine gun model and corresponding scene are loaded, the three-dimensional virtual demonstrate voluntarily ${ }^{[5]}$. So the subjects can learn the fault knowledge, understand the using and maintenance method. The subjects can choose the corresponding course interactive training operate of operate control module which can achieve the purpose of autonomous training. So the training people can be familiar with the using and maintenance operate directive rules after demonstrate. The learners will be examined after auto-training and the system will estimate the learners operate result according to the operate information ${ }^{[6]}$.

The interactive three-dimension virtual maintenance system is shown as fig. 2 according to the design thinking.

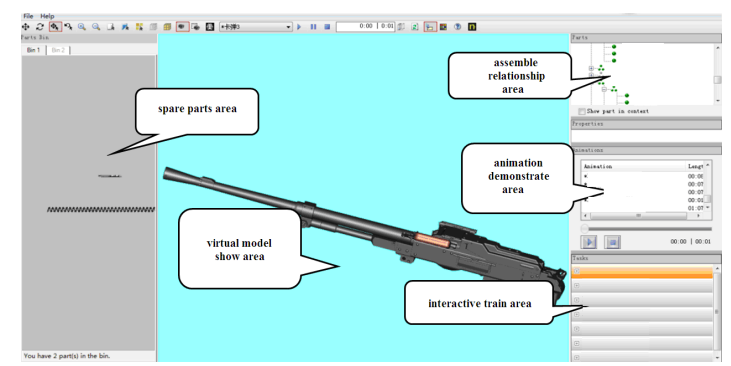

Fig.2 the three-dimension virtual maintenance system

The three-dimension virtual maintenance system is composed by five function areas. The assemble relationship area shows the construct and parts assemble relationships of parallel machine gun. The parts attribute area shows the name, number and simple description of other attributes. The three-dimension animation demonstration shows the animation of normative maintenance process to provide for the learners. The interactive maintenance training area can train and exam the leaners under the guidance of normative maintenance process. The replacement parts area is used to afford the replacement parts chosen. The parallel machine gun interactive virtual maintenance platform is built up according to the function area design which is shown as fig.3. 


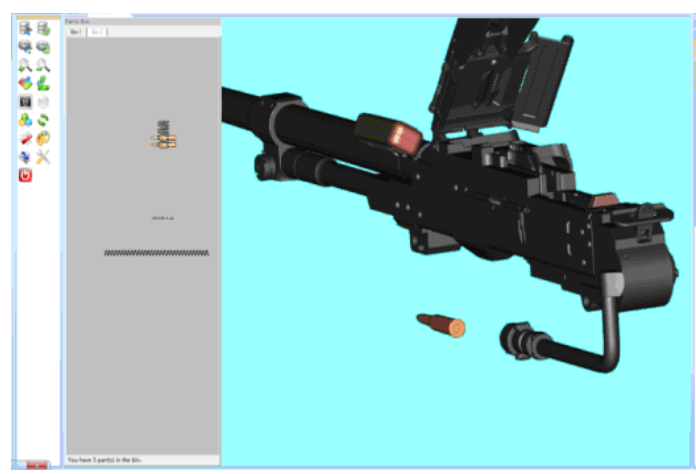

Fig. 3 the interactive virtual maintenance platform of parallel machine gun

\section{The PERT figure of maintenance training subject}

The PERT figure is built up to describe the parallel machine gun virtual maintenance process which is divided as maintenance training layer, fault phenomenon layer and maintenance work layer.

The maintenance training layer is named as the fault phenomenon afforded by fault simulation which is more close to the real maintenance training.

The fault phenomenon is used to describe the parallel machine gun fault phenomenon, the whole maintenance process simulation can be fulfilled according to the time sequence and normative basic maintenance work model.

The maintenance work layer can be divided as five type such as disassemble, assemble, diagnosis, change and verification according to the description.. the PERT figure is described according to the time sequence. The basic disassemble work is composed by a series real disassemble operations, the operation sequence is same with the parallel machine gun disassemble order. The basic test work is used to test the fault parts. The basic change work is used to take certain measures to recover the formulary function station after the target fault fixed. The basic assemble work is the opposite process of the disassemble work and the whole assemble work is the opposite process the whole disassemble work. The basic verification work is used to test the formulary function. 


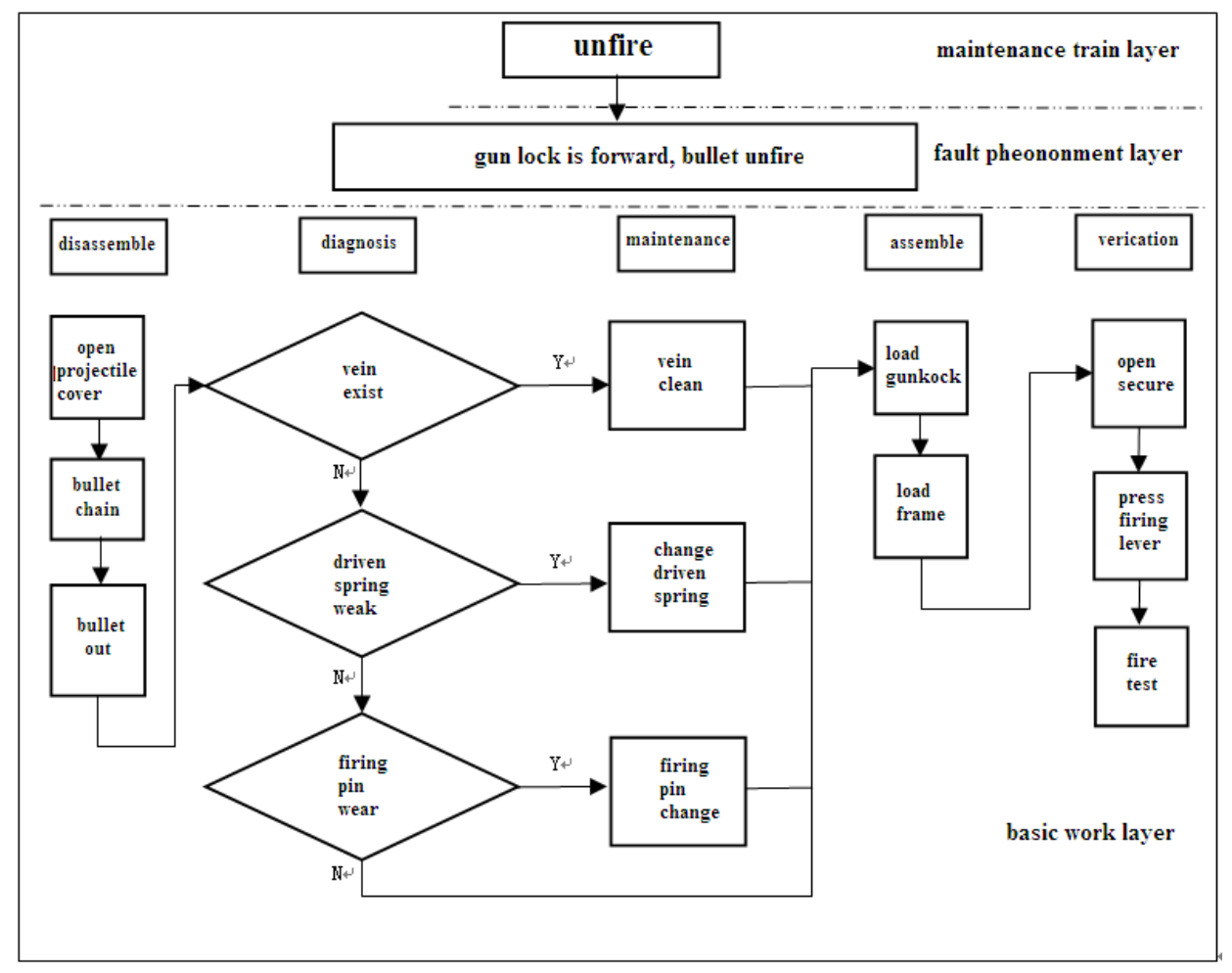

Fig.4 The PERT figure of unfired fault

The thinking of the whole parallel machine gun maintenance process is that according to the PERT figures of every fault phenomenon, simulate the basic operations such as disassemble, assemble, diagnose, change, repair, and verification, and every operation is independent. The learners can run the basic maintenance program script by human-computer interaction mechanism under the guidance of maintenance PERT figures. Then all the basic maintenance simulations can start-up according to the PERT figures and the whole maintenance process simulation can be fulfilled.

The interaction object can be the whole machine, subsystem, parts and components. The behavior can be the action such as move, revolve and prize. The interaction behavior will start-up after the condition is judged and the situation is fulfilled, the state marker will change when the action is finished. The interactive information flow path is shown as fig.5.

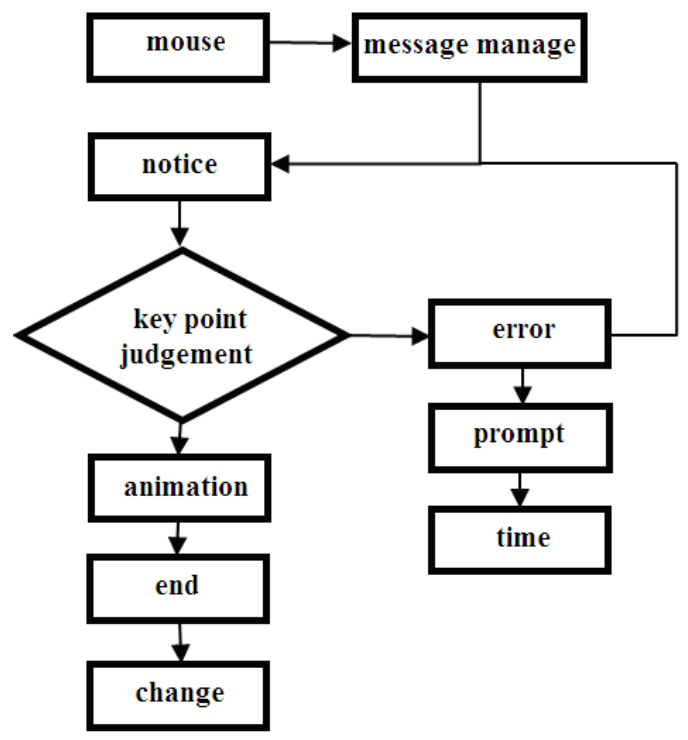

Fig. 5 the interactive information flow path 


\section{Interactive design of failure recovery}

The move and revolve operation are combined and some key frames are built up according to the orders and movie rules in the disassemble and assemble operations. The animation of disassembling the gunlock is shown as fig.6.

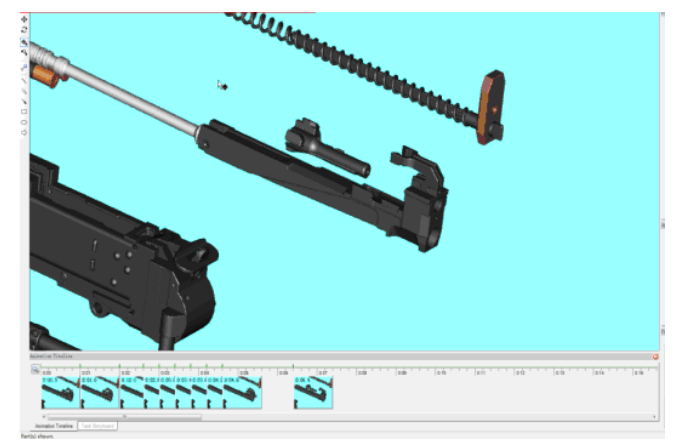

Fig.6 disassembling the gunlock

The task of removing the unfiring fault is built up and is shown as fig.7. The first key frame is to open the projectile cover and the key point is the bamboo shoot of the projectile cover. The animation will be played if success and the information of clicking the bamboo shoot of the projectile cover will be pointed out if failed. The second key frame is to take down the elastic chain and the key point is elastic chain. The animation will be played if success and the information of clicking the elastic chain will be pointed out if failed. The third key frame is to quit out the bullet from the chamber and the key point is the filling handle. The animation will be played if success and the information of clicking the filling handle will be pointed out if failed. Then the interactive function will finish according to the sequence.

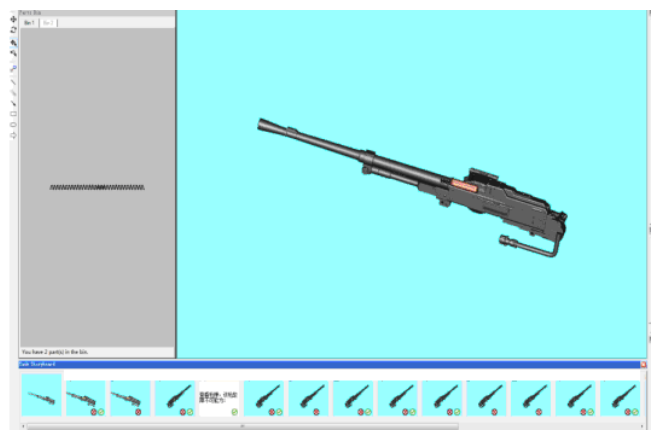

Fig.7 the task of unfired fault removing

The firing pin replacement part is changed and the learners push the firing pin replacement part to the original location and load it to the gunlock which is shown as fig.8.

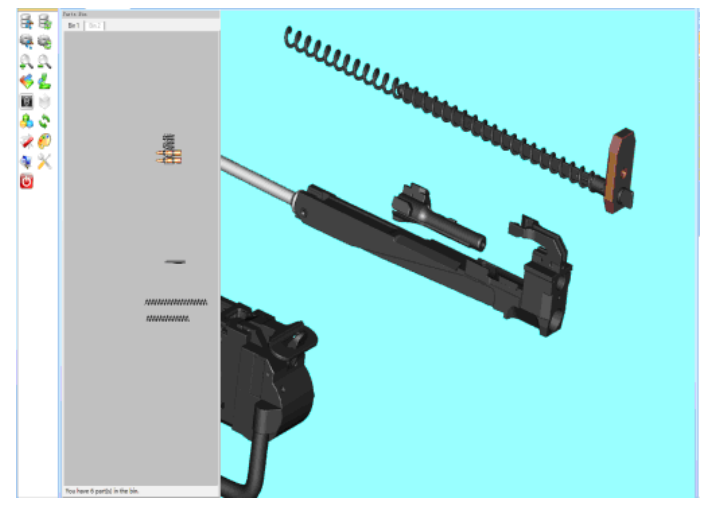

Fig. 8 change the firing pin replacement part 


\section{Summary}

This paper solved the problem of parallel machine gun three-dimension model building up and optimization in the virtual environment by designing interactive virtual maintenance training system using Ngrain software, finished the interactive virtual maintenance training of the parallel machine gun in the simulation environment based on the key points such as PERT figures of virtual maintenance process description, the basic maintenance work simulation of interactive behavior model and the interactive control of virtual maintenance operation flow path. The system has actual surface and flexible operability which separates the learners from the traditional sterile training mode and increases the maintenance training level.

\section{Reference}

[1] HaritosTom, Macchiarella, Nickolas D.A mobile application of augmented reality for aerospace maintenance training. AIAA / IEEE Digital Avionics Systems Conference-Proceedings, V 1, 24th DASC: 24th Digital Avionics Systems Conference-Proceedings, 2005:1563376 [2]ArendarskiBartlomiej, TermathWilhelm, MeckingPaul. Maintenance of complex machines inelectric power systems using Virtual Reality techniques.Conference Record of IEEE International Symposium on Electrical Insulation, 2008 IEEE International Symposium on Electrical Insulation, ISEI2008,2008:483-487

[3] Rosenberg, Barry. Lockheed martin demonstrates virtual maintenance software[J]. Aviation weeks \&space technology, 2002, 156 (14):89-93

[4] WhiskerVaughn E,BarattaAnthony J.Use of virtual environments to reduce the construction costs of the next generation nuclear power reactors . Societe Francaise d'Energie Nucleaire-International Congress on Advances in Nuclear Power Plants-ICAPP 2007, "The Nuclear Renaissance at Wbrk",V 4,2008: 2407-2416

[5] CHEN Di-jiang, ZHANG Hong-qi, CHEN Xing-yu. Research on High Efficient Virtual Maintenance Technology of Ground Based Radar Structure[J]. Radar Science and Technology,2010,18(6):576-579.

[6] ZHU xiao-jun, MIN shao-song, YUAN yum-ming, Research on Maintainability Virtual Verification System of Ship[J]. CHINA SHIP REPAIR, 2006 （12） : 40-42.

LI Hua (1978- ) , female, doctor, the main research direction is test and simulation. 